\title{
Academia-Industry Partnerships as Incubators for Economic Development
}

\section{Andrea Giuffrida*}

Director of Biomedical Research Development, Office of the Vice President for Research, Department of Pharmacology, University of Texas Health Science Center, San Antonio, TX 78229, USA

\section{Editorial}

The flattening of federal funding for biomedical research and the recent restructuring of the pharmaceutical industry have created a favorable momentum for collaborations between academia and the private sector.

Traditionally, academic medical centers have used clinical and scientific resources to foster pre-clinical and early clinical research, creating new platforms to translate discoveries at the bench into therapies. This mission, which has been framed and supported by the Bayh-Dole Act since the early 80s, currently faces several challenges that make translational research particularly troublesome. University administrators are balancing budgets nationwide by pulling physicianresearchers from the laboratory into medical offices in the attempt to increase clinical revenues. At the same time, the cuts in medical reimbursements have forced clinicians to pack their schedules with larger number of patients, leaving little time to collaborate with basic scientists, which are themselves overwhelmed by the daunting task of finding new funding sources and managing the growing administrative burden [1].

At the pharmaceutical industry level, the end of the blockbusterdrug era and the recent economic turndown has lead to a reorganization of research and development (R\&D) activities and to a significant downsizing of drug discovery, followed by massive layoffs and outsourcing [2]. In addition, the escalating costs of clinical trials, the toughening of evaluation criteria by the US Food and Drug Administration (FDA), and the limitations on patent exclusivity, have made the development of new therapeutics increasingly difficult and private investments too risky.

Within this dark scenario, new opportunities for growth are emerging not only for big pharmas, but also for the biotechnology industry and smaller size companies, which are ramping up their partnerships with academia with the intent to leverage the university workforce and refocus on "more promising" research areas. Pfizer, for example, is now actively working with academic medical hubs around Boston, New York, San Francisco and San Diego to set up centers for therapeutic innovation. In exchange, academic institutions are receiving conspicuous monetary investments and high-quality technical expertise in drug discovery. As recently pointed out by Robert Cailiff, Vice Chancellor at Duke University, these partnerships offer medical centers "a new key to diversify funding, and pay attention to efficiency and collaborate" [3].

The renewed interest in university/industry interactions is posing new challenges for the management of data sharing, conflict of interests and intellectual property. An interesting example is offered by the Sage Bionetworks, a non-profit medical research organization that promotes strategic public-private partnerships, organizes open-access scientific and clinical networks, and invites companies to share genetic and clinical data from patients enrolled in clinical trials [4]. Although this innovative approach provides valuable information to study disease mechanisms, it faces ethical and regulatory issues as the informed consents signed by patients do not allow the sharing of health data for scopes beyond those described in the original study. Thus, improving consent documents and processes to accommodate more flexibility will be crucial for the success of similar initiatives in the future.
University-industry collaborations may also prove to be valuable to avoid the so-called "valley of death", that stage of drug development where a promising experimental drug languishes and often dies before reaching the market. In this regard, the FDA has launched new guidelines to modernize regulatory science - the development and implementation of new tools, standards and approaches for assessing product safety, efficacy and quality - with the intent to foster public-private interactions and accelerate the translation of scientific discoveries into biomedical products [5]. These FDA initiatives have lead to the development of innovative in vitro models to test drug toxicity on organs on a chip [6]. If validated, these chips may allow bypassing whole-animal toxicological tests, and could be even personalized to detect individuals' drug responses. Thus, opening to regulatory science can be a smart investment for academia and health science centers as it represents a promising research avenue for which universities are already well positioned.

Although the ties to industry may offer alternative ways to academia to survive the shortage of public funding, the longevity of these interactions entails full transparency from all sides. How can we preserve academic freedom, autonomy, and publication rights when universities start to collaborate with industry? These issues are already encountered in all academic settings and often result, for example, in the delay of publications to accommodate patent filings or protect trade secrets. Nevertheless, the growing involvement of the private sector will require higher vigilance by faculty governing bodies to assess potential conflicts of interest, as well as the revision of current university policies to adopt new models to evaluate typical academic functions, such as faculty hiring/promotion, curriculum development, peer review, etc. In this context, the American Association of University Professors (AAUP) has drafted a report containing numerous recommendations to encourage new standards to guide sponsored research and more effectively manage individual and institutional financial conflicts of interest [7].

Challenges, however, are not limited to the points mentioned above, but also include other components of academic life, such as the training of students, residents and faculty. To invest more time on the development of biomedical products, scientists and clinicians should have access to educational resources to improve their entrepreneurial skills. An increasing number of schools are already offering master's degrees in translational science - in which physicians learn to become clinical researchers - and developing ties with local law schools and

*Corresponding author: Andrea Giuffrida, Director of Biomedical Research Development, Office of the Vice President for Research, Department of Pharmacology, University of Texas Health Science Center, San Antonio, TX 78229, USA, E-mail: giuffrida@uthscsa.edu

Received January 24, 2013; Accepted January 25, 2013; Published January 28 2013

Citation: Giuffrida A (2013) Academia-Industry Partnerships as Incubators for Economic Development. Pharmaceut Reg Affairs 2: e120. doi:10.4172/2167$7689.1000 \mathrm{e} 120$

Copyright: ( $) 2013$ Giuffrida A. This is an open-access article distributed under the terms of the Creative Commons Attribution License, which permits unrestricted use, distribution, and reproduction in any medium, provided the original author and source are credited. 
Citation: Giuffrida A (2013) Academia-Industry Partnerships as Incubators for Economic Development. Pharmaceut Reg Affairs 2: e120. doi:10.4172/2167-7689.1000e120

Page 2 of 2

business communities to orient researchers among the endless ways of technology transfer and the different regulatory pathways that medical devices, drugs and biologics should follow.

Finally, the success of future academia-industry partnerships should rely on a steady support from state and federal government. Guidelines on this matter can be found in a recent document released by the National Research Council (NCR), which provides recommendations to maintain the competitiveness of research universities and advance their contribution to national prosperity. In particular, tax incentives, investments to create physical spaces that support the day-to-day process of translation, and investments in cyber infrastructure, are all critical to build the bridges to connect laboratory-based scientists with clinicians and entrepreneurs, and ultimately improve university productivity and academic programs [8].

As the future of biomedical research is increasingly driven by multidisciplinary teams and by the need to interact with a variety of settings other than academia, initiatives that revitalize academia-industry partnerships will impact the economy beyond the geographical areas where they were originally conceived, and provide new tools to drive innovation and creativity.

\section{References}

1. Leshner Al (2011) Rethinking the Science System. Science 334: 738.

2. Pain E (2011) A Pharma Industry in Crisis.

3. Gura T (2012) US medical researchers brace themselves for budget cuts Lancet 380: 1371-1372.

4. Kaiser J (2012) Profile: Stephen Friend. The visionary. Science 335: 651-653.

5. Goodman JL (2012) Transforming Regulatory Science 2012: making a Difference. Clin Pharmacol Ther 91: 375-377.

6. Wenner Moyer M (2011) Organs-on-a-chip for Faster Drug Development Scientific American.

7. AAUP Report on Academic-Industry Partnerships, Washington DC.

8. Research Universities and the Future of America (2012) Ten breakthrough Actions Vital to our nation's Prosperity and security. The National Academy Press, Washington DC. 